\title{
Antimicrobial Susceptibility Testing of Metronidazole and Clindamycin against Gardnerella vaginalis in Planktonic and Biofilm Formation
}

\author{
Ting Li, ${ }^{1}$ Zhan Zhang, ${ }^{1}$ Fengjuan Wang, ${ }^{2}$ Yuanhui He, ${ }^{3}$ Xiaonan Zong, ${ }^{1}$ Huihui Bai, ${ }^{4}$ \\ and Zhaohui Liu $\mathbb{B}^{1}$ \\ ${ }^{1}$ Department of Gynecology, Beijing Obstetrics and Gynecology Hospital, Capital Medical University, Beijing 100026, China \\ ${ }^{2}$ Department of Obstetrics, Beijing Obstetrics and Gynecology Hospital, Capital Medical University, Beijing 100026, China \\ ${ }^{3}$ Department of Obstetrics and Gynecology, Peking University First Hospital, Beijing 100034, China \\ ${ }^{4}$ Department of Microecological Laboratory, Beijing Obstetrics and Gynecology Hospital, Capital Medical University, \\ Beijing 100026, China
}

Correspondence should be addressed to Zhaohui Liu; 17301255426@163.com

Received 3 March 2020; Accepted 25 May 2020; Published 16 June 2020

Academic Editor: Jorge Garbino

Copyright $\odot 2020$ Ting Li et al. This is an open access article distributed under the Creative Commons Attribution License, which permits unrestricted use, distribution, and reproduction in any medium, provided the original work is properly cited.

\begin{abstract}
Background. Bacterial vaginosis (BV), one of the most common vaginal ecosystem-related microbiologic syndromes, is the most common disorder in women of reproductive age. Gardnerella $(G$.) vaginalis is the predominant species causing this infection. Our aim was to compare the antimicrobial susceptibilities of metronidazole and clindamycin against G. vaginalis at planktonic and biofilm levels. Methods. From September 2019 to October 2019, we recruited a total of 10 patients with BV who underwent gynecological examinations at Beijing Obstetrics and Gynecology Hospital. G. vaginalis isolates were obtained from the vagina and identified using their characteristic colony morphology. Sequence data of clinical G. vaginalis isolates were confirmed by comparing $16 \mathrm{~S}$ rDNA sequences. Subsequently, clinical isolates were evaluated for antimicrobial susceptibilities in vitro to metronidazole and clindamycin at planktonic and biofilm levels. The minimum inhibitory concentration (MIC) for metronidazole and clindamycin was evaluated by antimicrobial susceptibility testing. The minimum biofilm eradication concentration (MBEC) was evaluated by the biofilm inhibition assay. Results. Planktonic clinical isolates showed a significantly higher susceptibility rate $(76.67 \%)$ and lower resistance rate $(23.33 \%)$ to clindamycin than to metronidazole (susceptibility rate: $38.24 \%$; resistance rate: $58.82 \% ; P<0.05$ for both). Furthermore, in comparison to planktonic isolates, the minimum inhibitory concentration (MIC) of metronidazole was significantly higher for biofilm-forming isolates $(7.3 \pm 2.6 \mu \mathrm{g} / \mathrm{mL} \mathrm{vs} .72 .4 \pm 18.3 \mu \mathrm{g} / \mathrm{mL}$; $P=0.005)$; the resistance rate was $27.3 \%$, and the minimum biofilm eradication concentration (MBEC) was $>128 \mu \mathrm{g} / \mathrm{mL}$. Moreover, the MIC of clindamycin was higher too for biofilm-forming isolates $(0.099 \pm 0.041 \mu \mathrm{g} / \mathrm{mL}$ vs. $23.7 \pm 9.49 \mu \mathrm{g} / \mathrm{mL}$; $P=0.034)$; the resistance rate was $27.3 \%$, and the MBEC of clindamycin was $28.4 \pm 6.50 \mu \mathrm{g} / \mathrm{mL}$. Conclusion. Our results indicate that in comparison to metronidazole, clindamycin seems to be a better choice to tackle G. vaginalis as it exhibits a relatively higher susceptibility rate and lower resistance rate.
\end{abstract}

\section{Introduction}

Bacterial vaginosis (BV) is one of the most common disorders of the lower genital tract in women of childbearing age. It represents an abnormal vaginal ecosystem, characterized by an initial decrease of healthy Lactobacillus-dominated vaginal microbiota and a subsequent increase of anaerobic and facultative bacteria such as Gardnerella (G.) vaginalis, Atopobium vaginae, and Prevotella bivia [1]. G. vaginalis has been isolated in up to 95\% cases, and it is the most typical and widely studied pathogen associated with $\mathrm{BV}$ [2]; the prevalence varies depending on race and ethnicity. For example, the $\mathrm{Na}$ tional Health and Nutrition Examination Survey 
2001-2004 reported that the prevalence of BV was $29.2 \%$ in women of reproductive age in the United States, and only $15.7 \%$ women with BV reported vaginal symptoms [3]. Furthermore, in a study conducted by Zhang et al. [4] on 1,218 married women in China, BV was the second most common disorder with an estimated prevalence of $10 \%$; in the Tibetan area of Sichuan Province, China, the prevalence of BV was reported to be $51.6 \%$ in a study conducted by Dai et al. [5]. BV can be considered a biofilm-associated infection, with a dense-structured polymicrobial biofilm consisting primarily of $G$. vaginalis adhering to the vaginal epithelium [6]. This biofilm sometimes ascends to the upper genital tract forming a polymicrobial endometrial and fallopian tube biofilm [7], and it may explain the associated with an increased risk of developing pelvic inflammatory diseases and adverse pregnancy outcomes, such as premature birth, abortion, and chorioamnionitis [1]. Moreover, BV is also associated with an increased risk of acquisition of sexually transmitted infections, such as human immunodeficiency virus [8] and Chlamydia trachomatis infection [9], and it may play a role as cofactors in human papillomavirusmediated cervical carcinogenesis [10]. The endometrial cavity is not sterile in most women with the presence of low levels of bacteria in the uterus, which is not associated with clinically significant inflammation [11]. However, the exact role of biofilm in relation to infectious diseases of the upper genital tract remains uncertain [12].

As the first-line of therapy for BV, the Centers for Disease Control and Prevention (CDC) recommends the use of oral or vaginally applied metronidazole or clindamycin [13]. Metronidazole, a derivative of nitroimidazole, is widely used. It may be administered orally at $500 \mathrm{mg}$ twice a day for 7 days or applied intravaginally in the form of a $0.75 \%$ gel once a day for 5 days [1]. Intravaginal application of clindamycin at bedtime for 7 days is also a common treatment regimen, but relapse can reportedly occur [1]. The relapse and recurrence of BV are the biggest challenges to current therapies; the recurrence rate is $>50 \%$ mainly because of the development of a multispecies biofilm, with G. vaginalis being one of the dominant species [14]. Nevertheless, both metronidazole and clindamycin have been reported to achieve cure rates of $70 \%-96 \%$, with recurrence rates of $49 \%$ $66 \%$; the relapse rates of metronidazole or clindamycin are quite high at $67 \%$ within 6-12 months [15]. The precise relationship between $\mathrm{BV}$-associated biofilmforming bacteria and treatment failure, however, still remains unknown. Accordingly, disrupting G. vaginalis biofilms seems to be a promising step toward developing a more sustainable way to treat $\mathrm{BV}$ and tackle its recurrence [16].

Many studies have reported the antimicrobial action of metronidazole and clindamycin on G. vaginalis, which is still considered to be the predominant bacteria causing BV. In the present in vitro study, we investigated the susceptibilities of planktonic G. vaginalis and biofilms to metronidazole and clindamycin and compared the antimicrobial abilities of these two antibiotics.

\section{Methods}

2.1. Ethical Approval of the Study Protocol. This study was approved by the Ethics Committee of Beijing Obstetrics and Gynecology Hospital, Beijing, China (2019-KY-030-01), and it was conducted in accordance with the principles expressed in the Declaration of Helsinki. All eligible participants provided written informed consent to be included in this study.

2.2. Patients and Sample Collection. From September 2019 to October 2019, we recruited a total of 10 patients with BV who underwent gynecological examinations at Beijing Obstetrics and Gynecology Hospital. The exclusion criteria included pregnancy, menstruation, sexual intercourse, or application of any intravaginal product within the last $24 \mathrm{~h}$, use of antibiotics in the last month, and lower genital tract malignancy. We also excluded women who had previously undergone cervical surgery or pelvic radiation therapy; those with Trichomonas vaginitis, vulvovaginal candidiasis, aerobic vaginitis, and other vaginal infections; and those allergic to metronidazole or clindamycin. Patients aged $18-50$ years and with a Nugent score of $\geq 7$ were included.

Regular gynecological examinations were performed, and vaginal secretions of patients with $\mathrm{BV}$ were sampled from the upper third of the vaginal wall using aseptic endocervical cotton swabs. We stained the smears using Gram's method; subsequently, the smears were examined by one observer at $\times 400$ magnification in an optical microscope (Leica Microsystems, Mannheim, Germany), and a Nugent score was assigned to each sample. Smears with a Nugent score of $\geq 7$ were considered to be diagnostic of $\mathrm{BV}$, while those with a score of 4-6 were diagnostic of intermediate BV, and $0-3$ were interpreted as normal flora [17].

2.3. Antimicrobial Preparation. Metronidazole was purchased from Fuzhou Haiwang Fuyao Pharmaceutical Co., Ltd (Fujian, China), and clindamycin palmitate was purchased from Guangzhou Yipinhong Pharmaceutical Co., Ltd (Guangdong, China). Metronidazole $(0.0625-64 \mu \mathrm{g} / \mathrm{mL})$ and clindamycin $(0.03125-32 \mu \mathrm{g} / \mathrm{mL})$ were stored as stock solutions, and to attain the desirable concentration, they were serially diluted using modified brain heart infusion (BHI) broth (Difco, Sparks, MD).

2.4. Identification and Isolation of G. vaginalis. 10 clinical isolates of $G$. vaginalis were identified using their characteristic colony morphology; representative colonies were selected from each vaginal secretion. We used Columbia blood agar base (Sigma-Aldrich, US), and colonies were isolated to purity. Gram stain showed that the isolates were Gram-variable pleomorphic rods, and the catalase reaction was negative. DNA from clinical G. vaginalis isolates and $A$. vaginae isolates was extracted using the QIAamp DNA Mini Kit (QIAGEN, Germany). For identifying the isolates, 16S rRNA gene hypervariable V1-V3 region was amplified using the primers 27F (5'-AGAGTTTGATCCTGGCTCAG-3') 
and 1492R ( $5^{\prime}$-GGTTACCTTGTTAGACTT-3'). Sequence data of clinical $G$. vaginalis isolates were confirmed by comparing $16 \mathrm{~S}$ rDNA sequences to the GenBank data library using the advanced gapped BLAST program. The purified isolates were stored at $-80^{\circ} \mathrm{C}$ in the De Man, Rogosa, and Sharpe (MRS) broth containing 30\% glycerol.

2.5. G. vaginalis and Planktonic Cultures. The clinical isolates were cultured using Columbia blood agar base (Becton Dickinson, Rockville, MD) at $37^{\circ} \mathrm{C}$ under anaerobic conditions in an anaerobic glove box (Coy Laboratory Products, Inc., Grass Lake, MI); the anaerobic chamber contained 5\% $\mathrm{H}_{2}, 5 \% \mathrm{CO}_{2}$, and $90 \% \mathrm{~N}_{2}$. Different isolates were plated onto the agar medium and then suspended in modified BHI broth using a $0.5 \mathrm{McF}$ arland standard, for a total concentration of $1.5 \times 10^{8} \mathrm{CFU} / \mathrm{mL}$, as previously described [18]. G. vaginalis ATCC ${ }^{\circledR} 14018$ was used as a control for the tests carried out under anaerobic conditions.

2.6. Biofilm Formation and Quantification. To develop the biofilm model of $G$. vaginalis, a starting inoculum of approximately $10^{6} \mathrm{CFU} / \mathrm{mL}$ of prepared bacterial suspension in the BHI broth with $0.4 \%(\mathrm{w} / \mathrm{v})$ glucose was added to different concentrations of metronidazole and clindamycin and inoculated into a 96-well microplate (Falcon, Corning Inc., Corning, NY) for $48 \mathrm{~h}$ at $37^{\circ} \mathrm{C}, 5 \% \mathrm{CO}_{2}$. The $\mathrm{BHI}$ media and biofilm culture without any compounds served as controls. Bacteroides fragilis ATCC $^{\circledR} 14018$ was used as a quality control bacterium and cultivated under anaerobic conditions. The plates were incubated for $48 \mathrm{~h}$, and the growth medium was replaced every $24 \mathrm{~h}$. Crystal violet staining (Sigma-Aldrich, St. Louis, MO, US) was used to quantify the total amount of biofilm biomass [19]. Biofilms were then analyzed after a total of $48 \mathrm{~h}$ of incubation at an optical density of $595 \mathrm{~nm}\left(\mathrm{OD}_{595}\right)$ using a microplate reader (Bio-Rad Laboratories, Hercules, CA, USA). The isolates were classified into four categories on the basis of their ability to form biofilms according to the cutoff $\mathrm{OD}$ value $(\mathrm{ODc})$, which was defined as three standard deviations (SD) above the mean $\mathrm{OD}$ of the negative control [20]: $\mathrm{OD} \leq \mathrm{ODc}$, no biofilm producer; $\mathrm{OD} \leq 2 \times \mathrm{ODc}$, weak biofilm producer; $\mathrm{OD} \leq 4 \times \mathrm{ODc}$, moderate biofilm producer; and $4 \times \mathrm{ODc}<\mathrm{OD}$, strong biofilm producer.

2.7. Antimicrobial Susceptibility Testing. Clinical G. vaginalis isolates were evaluated for antimicrobial susceptibilities in vitro to metronidazole and clindamycin (Sigma-Aldrich, St. Louis, MO, US) targeting planktonic cells; to achieve this, we used the anaerobic agar dilution method described by the Clinical and Laboratory Standards Institute (CLSI).

One-hundred microliters of the prepared bacterial suspension $\left(10^{6} \mathrm{CFU} / \mathrm{mL}\right)$ was added to different concentrations of metronidazole and clindamycin at $37^{\circ} \mathrm{C}$. After $48 \mathrm{~h}$, the bacterial growth was evaluated by taking an endpoint reading at $\mathrm{OD}_{595}$ with a microplate reader (Bio-Rad Laboratories, Hercules, CA, USA). The minimum inhibitory concentration (MIC) for metronidazole and clindamycin was defined as the lowest antibiotic concentration yielding marked reduction in the growth or no growth at all. Under the same conditions, G. vaginalis ATCC ${ }^{\circledR} 14018$ was tested using the broth microdilution assay. The microbiological susceptibility and resistant breakpoints for metronidazole $(<8 \mu \mathrm{g} / \mathrm{mL}$ and $\geq 32 \mu \mathrm{g} / \mathrm{mL})$ and clindamycin $(<2 \mu \mathrm{g} / \mathrm{mL}$ and $\geq 8 \mu \mathrm{g} / \mathrm{mL}$ ), as defined by CLSI, were used for interpreting MIC results [21].

2.8. Biofilm Inhibition Assay. Biofilms were used to evaluate the activity of metronidazole and clindamycin. After $48 \mathrm{~h}$ of incubation with antimicrobial-containing medium, biofilms were washed twice with sterile phosphate-buffered saline, dried, and stained with $0.2 \%$ crystal violet (Sigma-Aldrich); and the biofilms were solubilized in $100 \mu \mathrm{L}$ of $95 \%$ ethanol for $5 \mathrm{~min}$. The absorbance of the stain was measured to quantify the cell viability of each biofilm [18]. The minimum biofilm eradication concentration (MBEC) was defined as the lowest concentration of an antibiotic that completely inhibited the growth of microorganisms, indicating complete biofilm eradication, as previously described [22].

2.9. Statistical Analysis. Statistical Package for the Social Sciences v13.0 (SPSS Inc., USA) was used for statistical analysis. Results are presented as mean \pm SD of the mean of at least triplicates. The main test used was the $\chi^{2}$ test; $P \leq 0.05$ indicated statistical significance.

\section{Results}

During the study period, a total of 10 isolates of G. vaginalis were isolated. Clinical information for the patients was obtained as a routine laboratory practice in the study. The identity of all presumptively isolated G. vaginalis was confirmed by the PCR (Figure 1). The study population had a mean age of $37.7 \pm 11.03$ years, mean Nugent scores of $7.60 \pm 1.71$, and mean $\mathrm{pH}$ values of $4.69 \pm 0.40$. Five (50.0\%) of these were with abnormal vaginal discharge.

Biofilm formation by individual isolates was heterogeneous. 10 clinical $G$. vaginalis isolates and $1 G$. vaginalis isolate (ATCC ${ }^{\circledR} 14018$ ) could form biofilms: 5 (45.5\%) of these were weak biofilm producers, 5 (45.5\%) were moderate biofilm producers, and $1(9.0 \%)$ was a strong biofilm producer (Table 1).

In comparison to planktonic clinical isolates, the MIC of metronidazole was significantly higher for biofilm-forming isolates $(7.3 \pm 2.6 \mu \mathrm{g} / \mathrm{mL}$ vs. $72.4 \pm 18.3 \mu \mathrm{g} / \mathrm{mL} ; P=0.005$; Table 1 and Figure 2), indicating a significant correlation between resistance to metronidazole and the ability of $G$. vaginalis isolates to form biofilms. Six of the 11 biofilmforming isolates showed a high resistance to metronidazole, with a resistance rate of $54.5 \%$; MBEC was $>128 \mu \mathrm{g} / \mathrm{mL}$. On the basis of these results, the MBEC was not achieved with either metronidazole or clindamycin even at their highest concentrations. There was no significant linear correlation between the ability to form biofilms and the ratio of MIC values (i.e., ratio of the MIC value with and without biofilm 


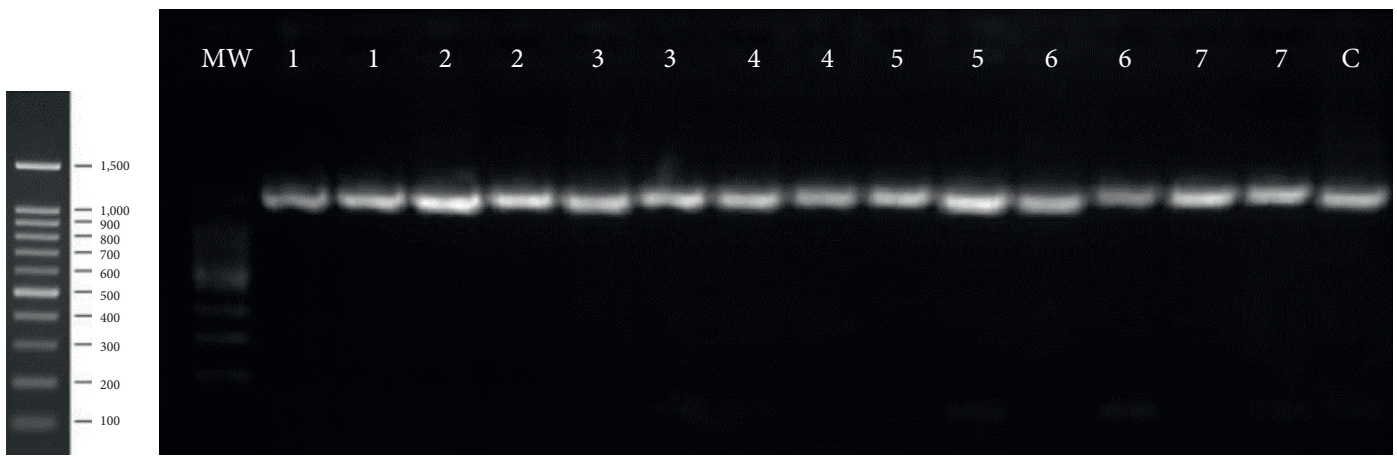

Figure 1: Representative electropherograms of Gardnerella vaginalis PCR identification. MW: molecular weight standard; lanes 1-7 in duplicate: bacteria isolated from vaginal secretions; Gardnerella vaginalis ATCC ${ }^{\circledR} 14018$ was the standard strain used as a quality control bacterium (C). Expected amplicon size: $1400 \mathrm{bp}$.

TABLE 1: Minimum inhibitory concentration (MIC) and minimum biofilm eradication concentrations (MBEC) for Gardnerella vaginalis at the planktonic and biofilm levels.

\begin{tabular}{|c|c|c|c|c|c|c|c|c|c|}
\hline \multirow[b]{2}{*}{ Isolate no. } & \multirow[b]{2}{*}{$\begin{array}{l}\text { Biofilm } \\
\text { producer }\end{array}$} & \multicolumn{4}{|c|}{ Metronidazole } & \multicolumn{4}{|c|}{ Clindamycin } \\
\hline & & $\begin{array}{c}\text { Planktonic MIC } \\
(\mu \mathrm{g} / \mathrm{mL})\end{array}$ & $\begin{array}{l}\text { Biofilm MIC } \\
(\mu \mathrm{g} / \mathrm{mL})\end{array}$ & $\begin{array}{l}\text { MIC } \\
\text { ratio }^{\text {a }}\end{array}$ & $\begin{array}{l}\text { MBEC } \\
(\mu \mathrm{g} / \mathrm{mL})\end{array}$ & $\begin{array}{c}\text { Planktonic MIC } \\
(\mu \mathrm{g} / \mathrm{mL})\end{array}$ & $\begin{array}{l}\text { Biofilm MIC } \\
(\mu \mathrm{g} / \mathrm{mL})\end{array}$ & $\begin{array}{l}\text { MIC } \\
\text { ratio }^{\text {a }}\end{array}$ & $\begin{array}{c}\text { MBEC } \\
(\mu \mathrm{g} / \mathrm{mL})\end{array}$ \\
\hline G1 & Moderate & 8 & 16 & 2 & $>128$ & 0.0312 & 1 & 32 & 32 \\
\hline G2 & Strong & 16 & $>128$ & 8 & $>128$ & $\leq 0.0312$ & $>64$ & 2048 & $>64$ \\
\hline G3 & Moderate & 2 & 8 & 4 & $>128$ & $\leq 0.0312$ & 0.5 & 16 & 8 \\
\hline G4 & Moderate & 2 & $>128$ & 64 & $>128$ & $\leq 0.0312$ & $>64$ & 2048 & $>64$ \\
\hline G5 & Moderate & 8 & $>128$ & 16 & $>128$ & $\leq 0.0312$ & $>64$ & 2048 & 32 \\
\hline G6 & Weak & 0.25 & $>128$ & 512 & $>128$ & $\leq 0.0625$ & 0.5 & 16 & $>64$ \\
\hline G7 & Weak & 2 & 0.5 & 0.25 & $>128$ & $\leq 0.0625$ & 0.5 & 16 & 32 \\
\hline G8 & Weak & 8 & $>128$ & 16 & $>128$ & 0.5 & 0.5 & 1 & 32 \\
\hline G9 & Weak & 2 & 4 & 2 & $>128$ & 0.125 & 0.5 & 4 & 32 \\
\hline G10 & Weak & $\leq 0.0625$ & $\leq 0.125$ & 2 & $>128$ & 0.125 & 1 & 8 & 8 \\
\hline $\begin{array}{l}\text { Standard } \\
\text { strain }^{\mathrm{b}}\end{array}$ & Moderate & 32 & 128 & 4 & $>128$ & $\leq 0.0625$ & 0.5 & 16 & 8 \\
\hline
\end{tabular}

MIC breakpoints adapted and interpreted as sensitive, resistant, or intermediate as defined by CLSI criteria for metronidazole (sensitive: $\leq 8 \mu \mathrm{g} / \mathrm{mL}$; intermediate: $=16 \mu \mathrm{g} / \mathrm{mL}$; resistant: $\geq 32 \mu \mathrm{g} / \mathrm{mL}$ ) and clindamycin (sensitive: $\leq 2 \mu \mathrm{g} / \mathrm{mL}$; intermediate: $=4 \mu \mathrm{g} / \mathrm{mL}$; resistant: $\geq 8 \mu \mathrm{g} / \mathrm{mL}$ ) were used for interpreting MIC results; The a MIC ratio was calculated using the following formula: MIC value with biofilm formation/MIC value without biofilm formation;

${ }^{\mathrm{b}}$ Gardnerella vaginalis ATCC ${ }^{\circledR} 14018$ was used as a quality control bacterium and cultivated under anaerobic conditions.

formation; mean value $4.0 \mu \mathrm{g} / \mathrm{mL}$, range $2.0-16.0 \mu \mathrm{g} / \mathrm{mL}$, $P=0.390)$.

As for clindamycin as shown in Table 1, the MIC of clindamycin was higher too for biofilm-forming isolates $(0.099 \pm 0.041 \mu \mathrm{g} / \mathrm{mL}$ vs. $23.7 \pm 9.49 \mu \mathrm{g} / \mathrm{mL} ; P=0.034)$; the resistance rate was $27.3 \%(3 / 11)$. Unlike metronidazole, there was a significant linear correlation between the ability to form biofilms and the ratio of MIC values (i.e., ratio of the MIC value with and without biofilm formation; mean value $16.0 \mu \mathrm{g} / \mathrm{mL}$, range $8.0-2048.0 \mu \mathrm{g} / \mathrm{mL}, P=0.025$, and correlation coefficient $=0.668$ ). The $\mathrm{MBEC}$ of clindamycin was $28.4 \pm 6.50 \mu \mathrm{g} / \mathrm{mL}$.

Our results indicated a significant difference between the MIC ratio of the two antibiotics with and without biofilm formation ( $P=0.083)$. The results for $G$. vaginalis showed that the tube MBEC/MIC ratio against metronidazole varied from 0.25 to 2048. The MBEC/MIC ratio against clindamycin varied from 64 to 1025, indicating significant differences in the effectiveness of two antibiotics in eradicating a biofilm population of two organisms.

\section{Discussion}

The BV recommended treatments are metronidazole and clindamycin, by the guidelines from the CDC and the American College of Obstetricians and Gynecologists (ACOG) $[13,23]$. However, the recommended treatments are still unsatisfactory, and high recurrence rates and resistance rates are frequent because the pathogenesis process involving $G$. vaginalis is not yet well understood. Biofilms are protective for the bacteria residing within as they can trap antibiotics before they reach their target [24], protect bacteria from the effects of the host immune system [25], and keep bacteria in a metabolically quiescent state induced by nutrient limitation inside the biofilm [26]. The central role of G. vaginalis in BV is attributed to its higher virulence potential [27] and ability to form biofilms that are stronger than those formed by other BV-associated bacteria $[28,29]$. Thus, G. vaginalis remains the primary pathogen of interest to study BV occurrence and recurrence. Efforts have been made to explore which microorganisms participate in the formation of a polymicrobial BV biofilm. In this study, we 


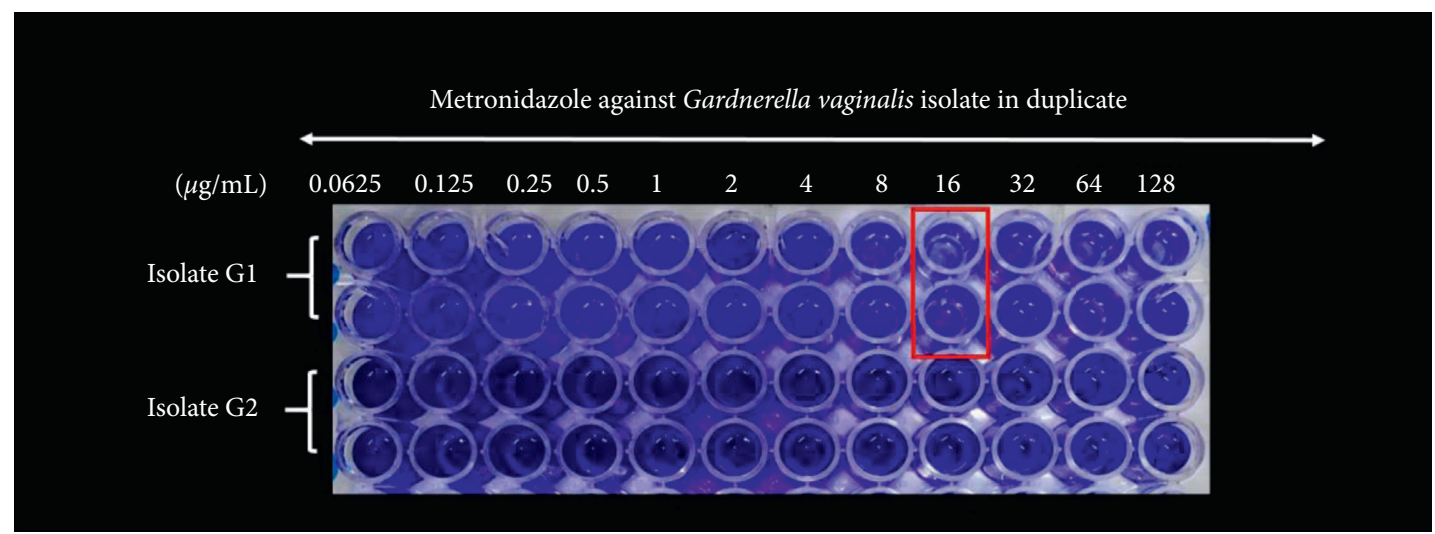

Figure 2: Biofilm formation testing by one clinical isolates of Gardnerella vaginalis (isolate nos. G1 and G2) against metronidazole. Clinical Gardnerella vaginalis (isolate nos. G1 and G2) was evaluated for antimicrobial susceptibilities in vitro to metronidazole targeting the biofilm. To develop the biofilm model, a starting inoculum of approximately $10^{6} \mathrm{CFU} / \mathrm{mL}$ of prepared bacterial suspension in the BHI broth with $0.4 \%(\mathrm{w} / \mathrm{v})$ glucose was added to different concentrations of metronidazole and inoculated into a 96 -well microplate for $48 \mathrm{~h}$ at $37^{\circ} \mathrm{C}, 5 \% \mathrm{CO}_{2}$. Crystal violet staining was used to quantify the total amount of biofilm biomass. Light blue wells with red box were considered as wells without growth, the minimum biofilm eradication concentration (MBEC), defined as the lowest concentration of an antibiotic that completely inhibited the growth of microorganisms, indicating complete biofilm eradication $(\mathrm{No} . \mathrm{G} 1: \mathrm{MBEC}=16 \mu \mathrm{g} / \mathrm{mL}$; No. G2: MBEC > $128 \mu \mathrm{g} / \mathrm{mL}$ ).

tested the ability of 10 clinical isolates of G. vaginalis to form biofilms; most of them were noted to be weak or moderate producers of biofilms in vitro.

$\mathrm{CDC}$ recommends that all symptomatic BV women should be treated using metronidazole and/or clindamycin [13]. Metronidazole, a first-generation nitroimidazole, is effective against anaerobes, but it has little or no activity against aerobes. BV-associated bacteria such as $G$. vaginalis is also sensitive to the hydroxymetabolite of metronidazole, which reportedly has a stronger antibiotic activity than the parent compound [30]. The metronidazole therapy is associated with several adverse effects such as nausea, vomiting, and gastrointestinal complaints [31]. Clindamycin, as a lincosamide, has anti-inflammatory properties with a broader range of activity against $\mathrm{BV}$ than metronidazole [32]. A previous study demonstrated that BV is a polymicrobial disorder that harbors particularly taxon-rich and diverse bacterial communities, which include a combination of not only anaerobic microorganisms but also other abnormal subtypes of mixed microorganisms found in women from China [33]. BV subtypes dominated by anaerobes can be more successfully treated using metronidazole, whereas clindamycin may be active against both metronidazolesensitive and other BV-associated strains with different microbial communities.

In a previous study, G. vaginalis isolates were reported to be susceptible to metronidazole and clindamycin with similar MIC values [34]. In this in vitro study, clindamycin showed a significantly higher susceptibility rate and lower resistance rate against planktonic clinical isolates than those shown by metronidazole. Compared to clindamycin, the susceptibility rate of clinical $G$. vaginalis isolates to metronidazole was significantly lower $(P=0.003)$. We found that biofilm's antibiotic administration equals to $\sim 2048 \mathrm{x}$ the MIC. Moreover, we found G. vaginalis biofilms to be highly tolerant to both metronidazole and clindamycin; this may create clinical challenges for obvious reasons. Surprisingly, a microbiologically significant reduction of biofilms could not be achieved with a clinically acceptable concentration of metronidazole for all biofilm-forming isolates, even for those with a weak biofilm-forming ability. With regard to treatment with clindamycin alone, coadministration of Lactobacillus preparations is recommended to help in the restoration of vaginal microenvironment. It is safe to assume that the high recurrence rate of $\mathrm{BV}$ is associated, at least in part, with the biofilm-formation ability of $G$. vaginalis. Due to the limited survey samples, a long-term and further study should be conducted to help us understand coculturing biofilm with Lactobacillus comprehensively and objectively, warranted to identify novel therapeutics that target vaginal biofilms in order to tackle both the occurrence and recurrence of $\mathrm{BV}$.

In summary, in comparison to metronidazole, clindamycin seems to be a better choice to tackle G. vaginalis; as according to our results, clindamycin showed a rather higher susceptibility rate and lower resistance rate at planktonic and biofilm levels. The recurrence of BV and antibiotic tolerance of BV-associated microorganisms may be associated with the ability of $G$. vaginalis isolates to form biofilms. Clinical $G$. vaginalis isolates that form biofilms can be hard to eradicate; therefore, further studies should be conducted with the aim of exploring new therapies that can be used in combination with currently known antibiotics, without affecting the normal vaginal flora, in order to prevent the formation of new biofilms and to eradicate the presence of persistent biofilms in patients with BV.

\section{Abbreviations \\ BV: Bacterial vaginosis \\ CDC: Centers for Disease Control and Prevention \\ BHI: Brain heart infusion}


RFLP: Restriction fragment length polymorphism

MRS: De Man, Rogosa, and Sharpe

ODc: Cut-off OD

SD: $\quad$ Standard deviations

CLSI: Clinical and Laboratory Standards Institute

MIC: Minimum inhibitory concentration

MBEC: Minimum biofilm eradication concentration.

\section{Data Availability}

The data used to support the findings of this study are included within the article.

\section{Conflicts of Interest}

The authors declare that there are no conflicts of interests regarding the publication of this paper.

\section{Acknowledgments}

This work was supported by a grant from the National Natural Science Foundation of China (grant nos. 81771530 and 81801406) and the Beijing Municipal Administration of Hospitals' Youth Programme (grant no. QML20181402).

\section{References}

[1] J. Paavonen and R. C. Brunham, "Bacterial vaginosis and desquamative inflammatory vaginitis," The New England Journal of Medicine, vol. 380, no. 11, pp. 1088-1089, 2019.

[2] D. N. Fredricks, T. L. Fiedler, and J. M. Marrazzo, "Molecular identification of bacteria associated with bacterial vaginosis," New England Journal of Medicine, vol. 353, no. 18, pp. 1899-1911, 2005.

[3] E. H. Koumans, M. Sternberg, C. Bruce et al., "The prevalence of bacterial vaginosis in the United States, 2001-2004; associations with symptoms, sexual behaviors, and reproductive health," Sexually Transmitted Diseases, vol. 34, no. 11, pp. 864-869, 2007.

[4] D. Zhang, T. Li, L. Chen et al., "Epidemiological investigation of the relationship between common lower genital tract infections and high-risk human papillomavirus infections among women in Beijing, China," PLoS One, vol. 12, no. 5, Article ID e0178033, 2017.

[5] Q. Dai, L. Hu, Y. Jiang et al., "An epidemiological survey of bacterial vaginosis, vulvovaginal candidiasis, and trichomoniasis in the Tibetan area of Sichuan Province, China," European Journal of Obstetrics, Gynecology, and Reproductive Biology, vol. 150, no. 2, pp. 207-209, 2010.

[6] L. Hardy, V. Jespers, N. Dahchour et al., "Unravelling the bacterial vaginosis-associated biofilm: a multiplex Gardnerella vaginalis and Atopobium vaginae fluorescence in situ hybridization assay using peptide nucleic acid probes," PLOS One, vol. 10, no. 8, Article ID e0136658, 2015.

[7] A. Swidsinski, H. Verstraelen, V. Loening-Baucke, S. Swidsinski, W. Mendling, and Z. Halwani, "Presence of a polymicrobial endometrial biofilm in patients with bacterial vaginosis," PLoS One, vol. 8, no. 1, Article ID e53997, 2013.

[8] C. Chehoud, D. J. Stieh, A. G. Bailey et al., "Associations of the vaginal microbiota with HIV infection, bacterial vaginosis, and demographic factors," AIDS, vol. 31, no. 7, pp. 895-904, 2017.
[9] C. T. Laughlin, E. K. Wurapa, W. B. Sateren, S. M. Morris, B. P. Hollingsworth, and J. L. Sanchez, "Association of bacterial vaginosis with chlamydia and gonorrhea among women in the U.S. army," American Journal of Preventive Medicine, vol. 52, no. 5, pp. 632-639, 2017.

[10] T. T. Suehiro, N. Malaguti, E. Damke et al., "Association of human papillomavirus and bacterial vaginosis with increased risk of high-grade squamous intraepithelial cervical lesions," International Journal of Gynecological Cancer, vol. 29, no. 2, pp. 242-249, 2019.

[11] C. M. Mitchell, A. Haick, E. Nkwopara et al., "Colonization of the upper genital tract by vaginal bacterial species in nonpregnant women," American Journal of Obstetrics and Gynecology, vol. 212, no. 5, pp. 611.e1-611.e9, 2015.

[12] C. S. Bradshaw and J. D. Sobel, "Current treatment of bacterial vaginosis limitations and need for innovation," Journal of Infectious Diseases, vol. 214, no. Suppl 1, pp. S14-S20, 2016.

[13] K. A. Workowski and G. A. Bolan, "Sexually transmitted diseases treatment guidelines, 2015," Recommendations and Reports: Morbidity and Mortality Weekly Report, vol. 64, no. 33, p. 924, 2015.

[14] D. Machado, J. Castro, A. Palmeira-de-Oliveira, J. Martinezde-Oliveira, and N. Cerca, "Bacterial vaginosis biofilms: challenges to current therapies and emerging solutions," Frontiers in Microbiology, vol. 6, p. 1528, 2016.

[15] A. Javed, F. Parvaiz, and S. Manzoor, "Bacterial vaginosis: an insight into the prevalence, alternative treatments regimen, and its associated resistance patterns," Microbial Pathogenesis, vol. 127, pp. 21-30, 2019.

[16] C. Gottschick, S. P. Szafranski, B. Kunze et al., "Screening of compounds against Gardnerella vaginalis biofilms," PLoS One, vol. 11, no. 4, Article ID e0154086, 2016.

[17] R. P. Nugent, M. A. Krohn, and S. L. Hillier, "Reliability of diagnosing bacterial vaginosis is improved by a standardized method of Gram stain interpretation," Journal of Clinical Microbiology, vol. 29, no. 2, pp. 297-301, 1991.

[18] Y. Turovskiy, T. Cheryian, A. Algburi et al., "Susceptibility of Gardnerella vaginalis biofilms to natural antimicrobials subtilosin, $\mathcal{\varepsilon}$-poly-L-lysine, and lauramide arginine ethyl ester," Infectious Diseases in Obstetrics and Gynecology, vol. 2012, Article ID 284762, 9 pages, 2012.

[19] S. Stepanovic, D. Vukovic, I. Dakic, B. Savic, and M. SvabicVlahovic, "A modified microtiter-plate test for quantification of staphylococcal biofilm formation," Journal of Microbiological Methods, vol. 40, no. 2, pp. 175-179, 2000.

[20] S. Stepanović, D. Vuković, V. Hola et al., "Quantification of biofilm in microtiter plates: overview of testing conditions and practical recommendations for assessment of biofilm production by staphylococci," APMIS: Acta Pathologica, Microbiologica, et Immunologica Scandinavica, vol. 115, no. 8, pp. 891-899, 2007.

[21] Clinical and Laboratory Standards Institute, Standards for Antimicrobial Susceptibility Testing, pp. M100-S26, Clinical and Laboratory Standards Institute, Wayne, PA, USA, 2016.

[22] R. K. Ruzicka and J. K. Struthers, "The use of Sorbarod biofilms to study the antimicrobial susceptibility of a strain of Streptococcus pneumoniae," Journal of Antimicrobial Chemotherapy, vol. 40, no. 4, pp. 601-602, 1997.

[23] J. A. Paavonen and R. C. Brunham, "Vaginitis in nonpregnant patients: ACOG practice bulletin, number 215," Obstetrics and Gynecology, vol. 135, no. 5, pp. 1229-1230, 2020.

[24] W. W. Nichols, S. M. Dorrington, M. P. Slack, and H. L. Walmsley, "Inhibition of tobramycin diffusion by binding to alginate," Antimicrobial Agents and Chemotherapy, vol. 32, no. 4, pp. 518-523, 1988. 
[25] Z. Xie, A. Thompson, T. Sobue et al., "Candida albicans biofilms do not trigger reactive oxygen species and evade neutrophil killing," Journal of Infectious Diseases, vol. 206, no. 12, pp. 1936-1945, 2012.

[26] P. Kashleva, T. Maira-Litran, A. J. McBain, A. H. Rickard, and F. W. Whyte, "The physiology and collective recalcitrance of microbial biofilm communities," Advances in Microbial Physiology, vol. 46, pp. 202-256, 2002.

[27] J. L. Patterson, A. Stull-Lane, P. H. Girerd, and K. K. Jefferson, "Analysis of adherence, biofilm formation and cytotoxicity suggests a greater virulence potential of Gardnerella vaginalis relative to other bacterial vaginosis-associated anaerobes," Microbiology, vol. 156, no. Pt 2, pp. 392-399, 2010.

[28] A. Machado and N. Cerca, "Influence of biofilm formation by Gardnerella vaginalis and other anaerobes on bacterial vaginosis," Journal of Infectious Diseases, vol. 212, no. 12, pp. 1856-1861, 2015.

[29] P. Alves, J. Castro, C. Sousa, T. B. Cereija, and N. Cerca, "Gardnerella vaginalis outcompetes 29 other bacterial species isolated from patients with bacterial vaginosis, using in an in vitro biofilm formation model," The Journal of Infectious Diseases, vol. 210, no. 44, pp. 593-596, 2014.

[30] C. S. Bradshaw, A. N. Morton, J. Hocking et al., "High recurrence rates of bacterial vaginosis over the course of 12 months after oral metronidazole therapy and factors associated with recurrence," The Journal of Infectious Diseases, vol. 193, no. 11, pp. 1478-1486, 2006.

[31] J. R. Garland, J. Marrazzo, A. P. Beelen, and J. D. Sobel, "A phase 3, multicenter, randomized, double-blind, vehiclecontrolled study evaluating the safety and efficacy of metronidazole vaginal gel $1.3 \%$ in the treatment of bacterial vaginosis," Sexually Transmitted Diseases, vol. 42, no. 7, pp. 376-381, 2015.

[32] A. Ianaro, A. Ialenti, P. Maffia et al., "Anti-inflammatory activity of macrolide antibiotics," The Journal of Pharmacology and Experimental Therapeutics, vol. 292, no. 1, pp. 156-163, 2000.

[33] Q. Di Rosa, L. Cheng, H. Zhang et al., "Identification of vaginal bacteria diversity and its association with clinically diagnosed bacterial vaginosis by denaturing gradient gel electrophoresis and correspondence analysis," Infection, Genetics and Evolution, vol. 44, pp. 479-486, 2016.

[34] M. A. B. Sun, L. A. Cosentino, L. K. Rabe, and S. L. Hillier, "Susceptibility of bacterial vaginosis (BV)-associated bacteria to secnidazole compared to metronidazole, tinidazole, and clindamycin," Anaerobe, vol. 47, pp. 115-119, 2017. 